\title{
On-Demand Service-Oriented Architecture and Standardization
}

\author{
Fei He \\ International Software School \\ Wuhan University, Wuhan, China \\ hefei.kh@whu.edu.cn
}

\author{
Keqing He, Peng Liang, Jian Wang \\ State Key Lab of Software Engineering \\ Wuhan University, Wuhan, China \\ hekeqing@sklse.org
}

\begin{abstract}
With the increased number of richness service resources, and maturity of (Service Oriented Architecture) SOA-based solutions, it is critical to develop an innovative software engineering methodology for user-centric and ondemand service oriented computing. This paper proposes the RGPS (Role-Goal-Process-Service) requirements metamodeling framework for service requirements elicitation, analysis, and modeling. Meanwhile, the standardization on service interoperability is a key driver to realize on-demand services based on various service resources. We have developed the ISO standards series: MFI (meta-model framework for interoperability) and MFI (Ontology-RGPS). We combine these two meta-model frameworks, which provide a foundation to bridge the gap from SOA to SSOA (Semantically-aware SOA), and further to On-Demand SOA.
\end{abstract}

Keywords: On-Demand Service, Software Virtualization, Metamodel Framework, Interoperability

\section{INTRODUCTION}

The ways of publishing and using software are experiencing dramatic changes. Software technologies, especially for service related software development, are facing a more complex and interactive application environment. The service software industry has a pressing need of driving forces from IT such as Service Oriented Architecture (SOA), and Software as a Service (SaaS) for considerable promotion of its innovation process. However, IT (software/hardware) and IT-based information management disciplines are still grounded on traditional knowledge systems; and there is no systematic approach to fill the huge gap within current IT fields: the services.

To address the challenges, we propose a framework with four core elements extracted from stakeholders' demands for services: Role, Goal, Process, and Service, which are named as RGPS requirements meta-modeling framework [1]. Meanwhile, the standardization on service interoperability is a key driver to realize on-demand services based on various service resources. To address this issue, this paper briefly introduces our works on Meta-model Framework for Interoperability (MFI) and its applications for service interoperability using RGPS framework as ISO standards. By combining these two meta-model frameworks, we propose the methodology of ODSOM (On-Demand ServiceOriented Modeling) to enable soft supporting for service virtualization, and its user-centric feature provides the capability of active on-demand service selection, which is able to support ODSOA (On-Demand SOA). We argue that the integration of RGPS framework and MFI can act as new standards for promoting the development of SaaS and cloud service.

\section{RGPS FRAMEWORK FOR SERVICE REQUIREMENTS MODELING}

We proposed and defined the RGPS requirements metamodeling framework, which acts as a bridge to realize the loosely-coupled connection and accurate matching between service requesters and providers [1]. To support service requirements modeling, we analyzed a large number of stakeholders' needs, with a special focus on their characteristics, including individuality, diversity, heterogeneity, uncertainty, etc. Based on the analysis and literature review, we proposed the RGPS (Role-GoalProcess-Service) requirements meta-modeling framework [1]. For instance, the role meta-model describes the social attributes (responsibility, organization, and rules) of stakeholders, the preferences of the stakeholders (i.e., actor), and situational constraints (e.g., a stakeholder could either be a user or a service). There are eight relationships defined among the four meta-models (R, G, P, and S).

To realize on-demand service, we use RGPS requirements meta-modeling framework to perform customized modeling of domain-oriented service aggregation with the support of Ontology-RGPS (O-RGPS). This customized modeling can facilitate reuse of domainoriented and large-grain service resources, and provide active service provisioning for personalized requirements from stakeholders.

RGPS meta-modeling framework has been applied in on-demand service systems development by several organizations with promising results, including the Department of Electronic Engineering, Tsinghua University; Internet-based Software Engineering Lab, Institute of Software, the Chinese Academy of Sciences; and Advanced Research Lab, Institute of Computing Technology, the Chinese Academy of Sciences.

\section{ON-DEMAND SOA (ODSOA)}

By investigation of recent studies of IBM's research efforts on service solutions and practices, we identify some points in common and complementary between IBM's S3 (Service-oriented Solution Stack) and our RGPS/MFI based ODSOM.

S3 (a service-oriented reference architecture across nine layers that aim to reinforce business value) [2] is a set of services or service components needed to deliver a fully functional solution. For example, to develop a Web application, the designer needs to use an operating system, Web server, database, and programming language. S3 assumes that a functional service requirement has two views. The provider view is the business and technical 
capability that a service must deliver to satisfy its consumers, while the consumer view is the business and technical capability that the service is expected to deliver in the context of that consumer alone.

The nine layers (operational systems, service component, services, business process, consumer, integration, QoS, information architecture, and governance and policies) are relatively independent, which allows the organization to choose the degree of consumer-provider integration. A SOA solution might exclude a business process layer, for example, have the consumer and service layers interact directly. Services are, of course, part of both consumer and provider views. The lower layers (services, service components, and operational systems) are provider concerns, while the upper layers (services, business processes, and consumers) are consumer concerns. More detailed explanation of each layer can be found in [2].
The discussion with lead researchers of IBM service research department makes us realize that we share several closer views towards SOA-based solution: i.e., consumer/business process/service view [3] vs. R/G/P/S view [1], in which consumer is a type of Role " $R$ " with specific business goals (a type of Goal " $G$ "), business process is a type of Process "P", and service element is equal between the two views. Both of us prefer and employ underlying meta-models (the model captures architectural building blocks). The layers in S3 represent different business value perspectives, which effectively separate concerns and offer considerable flexibility; this is quite match to the idea of service virtualization in our proposed ODSOM. Based on these findings, we propose the combination of S3 and Ontology-RGPS as an innovative on-demand solution of SOA, which is inline with methods such as ODSOMA, depicted in Figure 1.



Figure 1. The combination of S3 and O-RGPS (the red part embedded in S3 Logical layers)

\section{CONCLUSIONS}

This paper presents the ODSOM methodology and its ISO standardization on service interoperability management, which featuring user-centric, demand-oriented and interoperable services within service computing. Several practical applications using ODSOM have been successfully implemented and deployed in urban transportation system, and information systems of manufacturing industries, with tool support from RGPSbased service requirements modeling tool [4] to MFI(RGPS)-based service registration tool. This work has demonstrated a promising way for promoting the services capacity of SOA-based solutions, and improving the quality of customers' service experience. Compared with traditional SOA-based solutions that mostly address comparably stable user requirements, ODSOM can address the personalized and uncertain user requirements, which consequently improve the on-demand service capability and effectiveness.

\section{ACKNOWLEDGMENT}

This work is supported by the National Basic Research 973 Program of China under Grant No. 2007CB310801, the National Natural Science Foundation of China under Grant Nos. 60970017 and 60903034.

\section{REFERENCES}

[1] J. Wang and K.Q. He: RGPS: A Unified Requirements Meta-Modeling Frame for Networked Software, Proceedings of ICSE’08-IWAAPF, pages 29-35, 2008.

[2] A. Arsanjani, L-J. Zhang, M. Ellis, A. Allam, and K. Channabasavaiah: S3: A Service-Oriented Reference Architecture, IT Professional, 9(3):10-17, IEEE, 2007.

[3] A. Arsanjani and S. Ghosh: SOMA: A method for developing service-oriented solutions, IBM Systems Journal, 47(3):377-396, 2008.

[4] Z. Sun, J. Wang, K.Q. He, S. Xiang, and D. Yu: A Model Transformation Method in Service-Oriented Domain Modeling, Proceedings of the 21st Australian Software Engineering Conference (ASWEC), pages 107-116, 2010. 